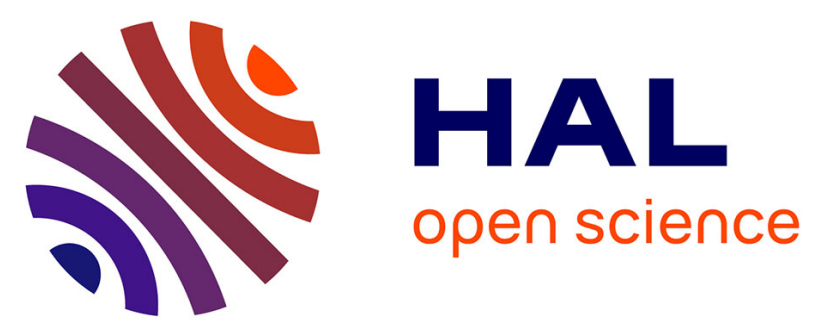

\title{
Multi-electron coincidence spectroscopy: Triple Auger decay of Ar 2p and 2s holes
}

P. Lablanquie, M. A Khalal, L. Andric, J. Palaudoux, F. Penent, J-M Bizau, D. Cubaynes, K. Jänkälä, Y. Hikosaka, K. Ito, et al.

\section{- To cite this version:}

P. Lablanquie, M. A Khalal, L. Andric, J. Palaudoux, F. Penent, et al.. Multi-electron coincidence spectroscopy: Triple Auger decay of Ar $2 \mathrm{p}$ and $2 \mathrm{~s}$ holes. Journal of Electron Spectroscopy and Related Phenomena, 2017, 220, pp.125 - 132. 10.1016/j.elspec.2017.04.003 . hal-01675125

\section{HAL Id: hal-01675125 \\ https://hal.sorbonne-universite.fr/hal-01675125}

Submitted on 4 Jan 2018

HAL is a multi-disciplinary open access archive for the deposit and dissemination of scientific research documents, whether they are published or not. The documents may come from teaching and research institutions in France or abroad, or from public or private research centers.
L'archive ouverte pluridisciplinaire HAL, est destinée au dépôt et à la diffusion de documents scientifiques de niveau recherche, publiés ou non, émanant des établissements d'enseignement et de recherche français ou étrangers, des laboratoires publics ou privés. 


\title{
Multi-electron coincidence spectroscopy: triple Auger decay of Ar $2 p$ and $2 s$ holes.
}

\author{
P. Lablanquie ${ }^{1}$, M. A. Khalal ${ }^{1}$, L. Andric ${ }^{1,2}, J_{\dot{5}}$ Palaudoux $^{1}$, F. Penent ${ }^{1}$, J-M Bizau ${ }^{3}$, D.

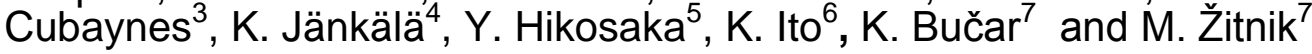

${ }^{1}$ Sorbonne Universités, UPMC Université Paris 06, CNRS, LCP-MR (UMR 7614), 11 rue Pierre et Marie Curie, 75231 Paris Cedex 05, France

${ }^{2}$ Université Paris-Est, 5 boulevard Descartes, F-77454 Marne-la Vallée Cedex 2, France ${ }^{3}$ ISMO, CNRS UMR 8214, Université Paris-Sud, Université Paris-Saclay, 91405 Orsay, France

${ }^{4}$ Nano and Molecular Systems Research Unit, University of Oulu, P.O. Box 3000, 90014 Oulu, Finland

${ }^{5}$ Graduate School of Medicine and Pharmaceutical Sciences, University of Toyama, 930-0194, Japan

${ }^{6}$ Synchrotron SOLEIL, I'Orme des Merisiers, Saint-Aubin, Boîte Postale 48, 91192 Gifsur-Yvette Cedex, France

${ }^{7}$ Jozef Stefan Institute, Jamova cesta 39, SI-1001 Ljubljana, Slovenia

\begin{abstract}
:
The detailed study of single photon multiple ionization processes requires the detection in coincidence of all the electrons emitted from the same ionization event. The advent of magnetic bottle experiments made possible the development of this multielectron coincidence spectroscopy. First we will briefly review the achievements of this technique. Then we will illustrate more specifically its high sensitivity taking as an example the decay by emission of three Auger electrons of $2 p$ and $2 s$ holes in argon. Our results show that the processes are completely different depending on the initial core hole: the three Auger electrons are emitted dominantly in a simultaneous path for the $2 p$ case, but in cascade for the 2 s one.
\end{abstract}




\section{Introduction}

Multi-electron coincidences are required to study in detail the single photon multiple ionization process:

$$
\mathrm{hv}+\mathrm{M} \rightarrow \mathrm{M}^{\mathrm{n}+}+\mathrm{ne} \mathrm{e}^{-}(1)
$$

In such a reaction, absorption of a single photon of energy hv by a molecule or an atom $M$ leads to the ejection of several $(n=2,3,4,5, \ldots)$ electrons. It is an interesting phenomenon forbidden in a simple model based on independent particles, that originates from electron-electron interactions. These multiple photoionization processes have thus attracted much interest as a direct probe of electron correlation. They are however often of low intensity and efficient experimental techniques are needed to study them.

Double photoionization $(n=2)$ has been widely studied with electron / electron coincidence techniques, and a large literature exists on $\mathrm{He}$ and $\mathrm{H}_{2}$ double photoionization, see for instance the reviews by Briggs and Schmidt [1] and Avaldi and Huetz [2]. Various coincidence techniques have been used to investigate double photoionization. One can cite electron-electron coincidences using two different electrostatic analyzers [1] [2], between two time-of-flight spectrometers [3] [4], using the TPEsCO (threshold photo-electrons coincidence) technique [5] [6] [7], using imaging microscopes such as the CIEL (Coïncidences entre lons et Electrons Localisés) apparatus [8] or the COLTRIM (COLd Target Recoil Ion Momentum) spectrometer [9]. The reader can refer to the very detailed and general review by Arion and Hergenhahn on coincidence spectroscopy [10]. Especially, angular correlations of the ejected electrons and molecular frame angular distributions have been obtained both for valence double ionization and for inner shell ionization followed by Auger effect. However with these techniques, studies of multiple ionization where more than 2 electrons $(n>2$ in (1)) are released have been limited to the observation of only two out of the $n$ ejected electrons [4] [11].

An important progress was achieved with the development in 2003 of the magnetic bottle spectrometer by J. Eland [12] [13], from an original concept presented by Beamson et al in 1980 [14] and implemented for laser experiments by Kruit and Read [15]. The advantage of this new spectrometer is to collect nearly all electrons emitted over the full $4 \pi$ solid angle and to measure their kinetic energy with a high resolution, better than that obtained with the COLTRIM or CIEL spectrometers. Direct angular information is lost, but would anyway be hardly exploitable as the degrees of freedom already amount to 9 when one wants to observe the kinetic energy and emission angle of 3 electrons. With these magnetic bottle spectrometers, it is now possible to detect in coincidence all the $n$ electrons emitted in a multiple photoionization process (1) and to study the energy correlations between them, opening new fields of investigations. Without trying to be exhaustive, we will cite here some important achievements made possible by the use of magnetic bottle spectrometers. They were initially obtained with a VUV laboratory source (pulsed He discharge lamp) and the first results were the retrieval of the complete valence double ionization spectra, both in atoms [13] and in molecules [12].

Implementation of magnetic bottles on synchrotron sources expanded the range of possible excitation energies and photoionization processes. The first achievement was the detailed study of the double Auger decay of a $4 d$ inner-shell hole in a Xe atom 
[16] making it possible to observe the $\mathrm{Xe}^{3+}$ final states which are populated, and the decay pathways, by cascade or simultaneous emission of the two Auger electrons. This work was followed by a number of studies of the double Auger decay of inner shell vacancies, both in atoms [17] [18] [19] [20] and in molecules [21] [22] [23]. The new multi-electron coincidence technique gave also access to the core valence double photoionization process, which, before that, had only observed as a 'shake off' continuum in photoelectron spectra, above the limit of satellite 'shake up' states. This was demonstrated by Hikosaka et al [24] in the case of the $\mathrm{Ne}$ atom and $\mathrm{N}_{2}$ molecule. The core valence double ionization of molecules has been the subject of many studies, with the aim of identifying the spectroscopy of the core-valence doubly charged species [25] [26] [27] [28]. As for atoms, it was recently demonstrated, with argon as an example [29], that these core valence double ionization experiments give access to the Auger spectra and inner shell levels of the associated ion; in other words it can be seen as an alternative to study inner shell photoelectron spectroscopy of an ion. Direct electron spectroscopy experiments on ions are extremely difficult and have only been reported up to now in a few cases, such as the $4 \mathrm{~d}$ photoionization of $\mathrm{Xe}^{+}$ions [30]; see also [31] and references included. Inner shell ionization of Krypton [32] and Mercury ions [33] [34] have recently been studied with this technique. Another field that could be explored thanks to magnetic bottle experiments is the triple photoionization process and the energy correlation between the three photoelectrons, either emitted from the valence shell [35] [36] or in a core-valence-valence triple photoionization path [37]. One must also mention the discovery of a source of low energy electrons in water clusters through the non-local ICD (Inter molecular Coulombic Decay) process, which was possible thanks to a magnetic bottle set up [38].

A new field that has recently experienced a large development thanks to the magnetic bottle capacities, is single photon double core photoionization. See for instance the recent review by Lablanquie et al [39]. Focus has been put especially on the process where the double core holes $(\mathrm{DCH})$ are created in a K-shell, as such states need to be populated by direct double photoionization and cannot be accessed by Auger decay of a deeper inner shell (contrary to $L^{-2}$ double core holes, for instance). Thanks to the coincidence detection of the two core photoelectrons and of the two subsequently released Auger electrons, it has been possible to observe double core holes in the same atom (1-site DCHs or $\left.\mathrm{K}^{-2}\right)$ [40] and also in different atoms of the molecule (2-site DCHs or $\left.\mathrm{K}^{-1} \mathrm{~K}^{-1}\right)$ [41]. One should note here the sensitivity of the multielectron coincidence technique, which is capable of detecting and studying the details of such weak double photoionization processes: the probability of 1-site and 2-site $\mathrm{DCH}$ formation amounts typically to $10^{-3}$ and $10^{-5}$, compared to the dominant single core hole $\left(\mathrm{K}^{-1}\right)$ formation. An extensive investigation of the spectroscopy of these $\mathrm{DCH}$ states [42] [43] [44] [45] [46], as well of their Auger decay paths [42] [47] followed. More recently interest has been drawn on the process (noted $\mathrm{K}^{-2} \mathrm{~V}$ ) where a photon simultaneously ionizes a core shell electron and excites another one from the same shell, motivated by the interesting spectroscopic properties of such states [48] [49] [50] [51].

In this manuscript we choose a simple example to illustrate the high performance and the sensibility of the multi-electron coincidence spectroscopy based on the use of a magnetic bottle: the triple Auger decay of a $2 p$ hole in the argon atom. This process will be compared to the triple Auger decay of the argon $2 \mathrm{~s}$ hole, we have examined previously [52]. The argon $2 p$ hole is known to decay predominantly by emission of a 
single Auger electron, and to a lesser extent by emission of two Auger electrons [4] [53]. But decay by emission of three Auger electrons is also possible, although it is a very weak process with a $0.18 \%$ probability [53]. Nevertheless we will see that it is possible to study this process in great detail. The surprising result is that the emission of the three Auger electrons is mainly simultaneous, contrary to the case of the triple Auger decay of the 2 s hole which proceeds dominantly by cascade [52].

\section{Experiment}

Our HERMES (for High Energy Resolution Multi Electron Spectrometer) experiment has been described in detail previously. See for instance references [18] [48] [54] Briefly, it is an adaptation, for use with synchrotron radiation, of the magnetic bottle time of flight spectrometer developed by J. Eland [13]. The magnetic fields in the spectrometer results from the superposition of the strong magnetic fields of a ( 5000 Gauss) SmCo (or NdFeB) permanent magnet and a weak field (10Gauss) of a $2 \mathrm{~m}$-long solenoid. This creates a magnetic mirror configuration for the source volume, and all electrons produced in the multiple photoionization of a single atom or molecule by a pulse of light from the synchrotron, are collected and guided through the solenoid towards an MCP (Micro Channel Plate) detector. In the present version, the individual time of flight of electrons, with respect to the light pulse, are recorded by a Time to Digital Converter with a 120ps discretization step ('TDC-V4' developed at the LUMAT federation in Orsay, France). Calibration and conversion from electron time of flights to kinetic energies were achieved by measuring photoelectron lines of helium and argon at different photon energies. The dead time for the detection of two successive electrons was reduced here to $4 \mathrm{~ns}$. The energy resolution $\Delta \mathrm{E} / \mathrm{E}$ of the spectrometer was $1.6 \%$ for $\mathrm{E}>1 \mathrm{eV}$. The electron detection efficiencies were estimated from photoelectron / Auger electron coincidences and were found to amount here to $52 \pm 5 \%$ for electron kinetic energies up to $200 \mathrm{eV}$.

The experiments on argon were carried out at the SOLEIL synchrotron facility in Saint Aubin (France), on the undulator beamline PLEIADES [55]. Single bunch operation of the SOLEIL synchrotron provided light pulses every 1184ns. For the spectra presented here, a $\sim 12 \mathrm{~h}$ accumulation was used with electron count rates of $\sim 15$ $\mathrm{kHz}$. The data obtained at two photon energies of 640 and $650 \mathrm{eV}$ were combined here. These two distinct photon energies were used to eliminate the possible structures due to false coincidences.

\section{The triple Auger decay of an Ar $2 p$ hole}

Since its first measurement in the 1960s by Mehlhorn et al [56], the noncoincident $\mathrm{L}_{2.3} \mathrm{MM}$ Auger spectrum of argon has been the subject of many studies, as shown by Pulkkinen et al [57] and references therein. The decay of $2 p$ holes by emission of two Auger electron was observed already in 1966 by Carlson and Krause [58]. Coincidence experiments brought more detailed information both for single [59] and double Auger decays, revealing for instance angular correlation patterns of the two Auger electrons emitted in direct double Auger decay [4]. The complete 2p Auger decay paths were examined recently with our HERMES magnetic bottle [53], allowing the detailed observation of the direct and cascade processes present in the double Auger decay, and revealing the existence of a weak triple Auger decay path. We revisit here 
this process with much higher statistics. Figure 1 shows the single, double and triple Auger spectra associated to the $2 p$ hole decay. They have been obtained from the coincidence events where, respectively, at least 1, 2 or 3 electron(s) were detected in coincidence with a $2 p$ photoelectron. The double and triple Auger spectra are plotted as a function of the sum of the kinetic energies of the Auger electrons; their structure originates from the $\mathrm{Ar}^{\mathrm{n}+}$ final states populated in the Auger decays. These Auger spectra are in good agreement with the coincident ones obtained previously [53], but here the $2 p_{3 / 2}$ and $2 p_{1 / 2}$ components are not separated. From these data, and taking into account the estimated electron detection efficiencies, it is possible to extract the probability for a $2 p$ hole to decay by emission of $n$ Auger electrons. The result is summarized in Table 1. One finds values in good agreement with our previous estimate [53]. It is seen that the probability of decay by emission of three electron is extremely weak $(0.18 \%)$. Note that a decay by emission of four electrons is not possible, because the ground state $\mathrm{Ar}^{5+}$ level has a binding energy (309.83eV [60]) higher than the $\mathrm{Ar}^{+} 2 \mathrm{p}$ ionization threshold (248.63 and $250.78 \mathrm{eV}$ respectively for the $2 \mathrm{p}_{3 / 2}$ and $2 \mathrm{p}_{1 / 2}$ components [61]).

\begin{tabular}{|c|c|c|c|c|c|}
\hline \multirow{2}{*}{$\begin{array}{c}\text { Final ionic } \\
\text { charge }\end{array}$} & $\begin{array}{c}\text { Type of } \\
\text { Auger decay }\end{array}$ & $\begin{array}{c}2 \mathrm{p} \\
\text { (this work) }\end{array}$ & $\begin{array}{c}2 \mathrm{p}_{3 / 2} \\
{[53]}\end{array}$ & $\begin{array}{c}2 \mathrm{p}_{1 / 2} \\
{[53]}\end{array}$ & $\begin{array}{c}\text { Relative abundances }(\%) \\
{[52]}\end{array}$ \\
\hline+2 & Single & 90.3 & 90.4 & 90.7 & 3 \\
\hline+3 & Double & 9.5 & 9.4 & 9.1 & 89 \\
\hline+4 & Triple & 0.18 & 0.18 & 0.18 & 8 \\
\hline+5 & Quadruple & - & - & - & 0.3 \\
\hline
\end{tabular}

Table 1 : Relative abundances of multiply charged $\mathrm{Ar}^{\mathrm{n}+}$ ions produced by Auger decay of the argon $2 p$ and 2 s holes.

Figure 2 is an expanded view of the $2 p$ triple Auger spectrum plotted in the bottom of Figure 1. The statistics and background level have been much improved compared to our previous study (Figure 9 in [53]). It reveals clearly the relative intensities of the $\mathrm{Ar}^{4+}$ states which are reached in the $2 \mathrm{p}$ triple Auger decay. The experimental resolution, estimated to $\sim 1.5 \mathrm{eV}$, is due to combination of the energy resolutions on each of the Auger electron of the triplet. It is sufficient to show the fine structure due to the decay of the individual $2 p_{3 / 2}$ and $2 p_{1 / 2}$ components. Note that these are here well separated, demonstrating that it is not necessary to resolve the $2 p$ spinorbit components in order to study the detail of the $2 p$ triple Auger decay. One observes the $\mathrm{Ar}^{4+} 3 \mathrm{p}^{-4}\left({ }^{3} \mathrm{P}\right)$ and $\left({ }^{1} \mathrm{D}\right)$ states that are dominantly populated and also, in less proportions, the $\mathrm{Ar}^{4+} 3 \mathrm{p}^{-4}\left({ }^{1} \mathrm{~S}\right)$ and $\mathrm{Ar}^{4+} 3 \mathrm{~s}^{-1} 3 \mathrm{p}^{-3}\left({ }^{3} \mathrm{D}\right)$ states. A close look at Figure 2 suggests that the $\mathrm{Ar}^{4+} 3 \mathrm{~s}^{-1} 3 \mathrm{p}^{-3}\left({ }^{1} \mathrm{D}\right)$ and $\left({ }^{3} \mathrm{P}\right)$ excited states are also populated, although they have not been included in the fit.

The next step is to investigate the energy correlation between the three Auger electrons. This is a three dimensional problem, but it can be reduced to a two 
dimensional one if we impose that the sum of the energies of the three electrons is fixed. In this case energy correlation can be represented in a 2-dimensional plot. These plots are known as Dalitz plots [62] and have been widely used in nuclear physics and molecular dynamics studies. We have used a variant of Dalitz plots to represent energy correlations of 3 electrons of fixed total energy [52] [37]. It has been defined in reference [52]; briefly, a triplet of electrons with energies $\left(E_{1}, E_{2}, E_{3}\right)$ satisfying $E_{1}+E_{2}+E_{3}=E_{S}$ appears in an equilateral triangle of height $E_{S}$ at a point whose distances to the three sides of the triangles are given by the $E_{i}$ energies. The possible combinations for the choice of the order of the three energies imply that a given triplet contributes to 6 points in the Dalitz plot. Another important property is that the integration of the overall intensity by projection on a perpendicular height of the triangle gives the $E_{i}$ energy distribution of any of the three Auger electrons. In the present case the Dalitz plot of Figure 3 (left) shows the energy correlation between the three Auger electrons emitted upon $2 p$ hole decay, when a final $\mathrm{Ar}^{4+} 3 p^{-4}$ is reached (the energy sum of the three electrons is selected here in the [102-107.7eV] energy range). One observes a fairly smooth and uniform filling of the Dalitz triangle, with a random energy sharing between the 3 electrons, which suggests a direct process for the ejection of the three Auger electrons. Higher intensity is found in the corners of the Dalitz triangle in Figure 3, which correspond to release of an Auger electron of close to $0 \mathrm{eV}$. It is also the zone where false coincidences are expected to contribute most, because of the presence in our experiment of low energy, secondary electrons. Fig 3 (right) displays the energy distribution of one of the three Auger electrons in this case where a final $\mathrm{Ar}^{4+} 3 \mathrm{p}^{-4}$ state is reached. As explained above it is obtained by projection of the 2-dimensional Dalitz plot (Fig 3 left) on its perpendicular height. The black (dotted) curve gives an estimate of the background due to false coincidences, obtained by selecting an energy range [110$115.7 \mathrm{eV}]$ for the sum of the three electrons coincident with a $2 p$ photoelectron, where no $\mathrm{Ar}^{4+}$ states are energetically allowed. It is seen that the energy distribution for one Auger electron is smooth with a edge or ' 2 ' shape evoking the well-known ' $U$ ' shape for the energy sharing between 2 electrons ejected in a direct process. The striking results from this analysis is thus that the triple Auger decay of a $2 p$ hole is dominantly a simultaneous emission of the three correlated Auger electrons. This is surprising, because for the $2 p$ double Auger decay, the energy distribution of one electron is much more structured, demonstrating the importance of cascade double Auger decays (See Figure 6 and 7 in [53]). However there must exist indirect triple Auger decay processes corresponding to the paths in which excited $\mathrm{Ar}^{3+^{*}}$ states, converging to excited $\mathrm{Ar}^{4+}$ states evidenced in Fig. 2, and that are populated by direct or cascade double Auger decay, will decay to lower $\mathrm{Ar}^{4+}$ levels, by emitting a $3^{\text {rd }}$ Auger electron. In order to find evidence for such decay paths we analyzed in more details the low energy part of the energy distribution of one of the triple Auger electrons from Figure 3 (right). It is reproduced in Figure $4(\mathrm{~A})$. Figure $4(\mathrm{~B})$ is a replot of the same data but with a smaller bin size of $10 \mathrm{meV}$ instead of $100 \mathrm{meV}$. Statistics is decreased but structure in form of weak peaks lying on a decreasing continuum are observable. They are of much weaker relative intensities compared to the $2 p$ double Auger decay case [53], suggesting a dominant simultaneous emission for the $2 p$ triple Auger decay.

The reason for this difference between $2 p$ double and triple Auger decays can be found if we examine the $\mathrm{Ar}^{\mathrm{n}+}$ energy levels. See for instance Fig. 2 in Arp et al [63] (we have done extended energy level calculations that confirm these results): they show that there is no cascade path (if high satellite Rydberg states are omitted) from $\mathrm{Ar}^{+} 2 \mathrm{p}^{-1}$ to $\mathrm{Ar}^{4+}$ states. Ar $2 \mathrm{p}^{-1}$ is higher in energy than $\mathrm{Ar}^{4+}$ states, but if the atom decays from 
$2 \mathrm{p}^{-1}$ via single or double decay, these states are lower in energy than any of the $\mathrm{Ar}^{4+}$ states and therefore terminate the cascade, resulting in a lower charge state than $4^{+}$. On the opposite there are possible cascade paths from $\mathrm{Ar}^{+} 2 \mathrm{p}^{-1}$ to $\mathrm{Ar}^{3+}$ states, through excited $\mathrm{Ar}^{2+}$ states lying above $\mathrm{Ar}^{3+}$ threshold, such as the $\mathrm{Ar}^{2+} 3 \mathrm{~s}^{-2}$ correlation satellites states of $3 s^{2} 3 p^{2} 3 d^{2}$ configuration [53]. Recently Müller et al [64] observed a clear triple Auger decay case where the three Auger electrons are necessarily simultaneously emitted, during the decay of an excited $1 \mathrm{~s}^{-1} 2 \mathrm{~s}^{2} 2 \mathrm{p}^{2} \mathrm{C}^{+}$state. It would be interesting to measure the energy sharing of the three Auger electrons in that case, and to compare with the present argon $2 p$ decay.

\section{The triple Auger decay of an Ar 2s hole}

It is interesting to compare the triple Auger decay of the argon $2 p$ hole with that of the $2 \mathrm{~s}$ one. The complete Auger decay tree of the $2 \mathrm{~s}$ hole was reconstructed recently with our magnetic bottle experiment [52]. The probability for the decay of a $2 \mathrm{~s}$ hole by emission of three Auger electron was found to be $8 \%$ (see table I) which is more than an order of magnitude larger than in the $2 p$ case. Figure 5 shows the triple Auger spectrum for the $2 s$ decay, to be compared with the one in Fig 2 for the $2 p$ decay. The resolution is worse in this case because of the larger kinetic energy of the Auger electrons that is possible in the 2 s triple Auger decay, compared to the $2 p$ one, but also because of the short lifetime of the 2 s hole which induces a lifetime broadening of the Auger spectra of $2.25 \mathrm{eV}$ [65]. However Figure 5 demonstrates that, similarly to the $2 p$ case, it is the $\mathrm{Ar}^{4+} 3 \mathrm{p}^{-4}$ states (and mainly the $\mathrm{Ar}^{4+} 3 \mathrm{p}^{-4}\left({ }^{3} \mathrm{P}\right)$ one) that are formed preferentially, and to a lesser extend the $\mathrm{Ar}^{4+} 3 \mathrm{~s}^{-1} 3 \mathrm{p}^{-3}$ state.

The Dalitz plots for the triple Auger decay of the 2 s hole to the $\mathrm{Ar}^{4+} 3 \mathrm{p}^{-4}$ and $\mathrm{Ar}^{4+}$ $3 s^{-1} 3 p^{-3}$ states are represented respectively in Figure $6 \mathrm{~A}$ ) and $6 \mathrm{~B}$ ). They have already been reported (see Fig 7 in reference [52]), and the present data fully confirm the previous measurements. The higher statistics reveals more clearly the different processes, and the shorter dead time of the detector helps reduce the dead zones in the Dalitz plots where two electrons of close energies contribute. The Dalitz plots for the $2 s$ triple Auger decay are observed to be completely different from the ones showing the $2 p$ triple Auger decay in Figure 3. As demonstrated in [52], the 2s triple Auger decay proceeds mainly by cascade. In a first step a Coster Kronig Auger decay takes place in which a $2 p$ and a valence electron contribute, producing the emission of a first Auger electron and the two large bands at $\sim 45 \mathrm{eV}$ and $\sim 30 \mathrm{eV}$ in the spectrum of the energy of one of the Auger electrons (Fig 6, right). These bands correspond respectively to the formation of the intermediate $\mathrm{Ar}^{2+} 2 \mathrm{p}^{-1} 3 \mathrm{p}^{-1}$ and $\mathrm{Ar}^{2+} 2 \mathrm{p}^{-1} 3 \mathrm{~s}^{-1}$ states. In a second step the $2 p$ holes in these intermediate states will evolve by double Auger decay. The energy sharing between these last two Auger electrons appears e.g. in the horizontal bands of the Dalitz plots, at the corresponding energy positions for the broad $\sim 45$ and $\sim 30 \mathrm{eV}$ bands in Figure 6 . Both a cascade path (large structured intensities on the edges) and a direct path (weak smooth intensity all along the lines) are observed for the emission of these last two Auger electrons. It is interesting to note that the direct release of the last 2 Auger electrons is more intense for the decay of the $\mathrm{Ar}^{2+} 2 \mathrm{p}^{-1} 3 \mathrm{p}^{-1}$ intermediate state to 
the $\mathrm{Ar}^{4+} 3 \mathrm{p}^{-4}$ final one (compared to the decay to the $\mathrm{Ar}^{4+} 3 \mathrm{~s}^{-1} 3 \mathrm{p}^{-3}$ final state), as seen in Fig $6 \mathrm{~A}$ ); and for the decay of the $\mathrm{Ar}^{2+} 2 \mathrm{p}^{-1} 3 \mathrm{~s}^{-1}$ intermediate state to the $\mathrm{Ar}^{4+} 3 \mathrm{~s}^{-1} 3 \mathrm{p}^{-3}$ final state, as seen in Fig $6 \mathrm{~B}$ ). In other words the $\mathrm{v}^{-1}$ valence hole of the $\mathrm{Ar}^{2+} 2 \mathrm{p}^{-1} \mathrm{v}^{-1}$ intermediate state prefers to remain spectator of the direct double Auger decay of the $2 p$ core hole.

\section{Conclusion}

In conclusion we have presented our multi-electron coincidence spectroscopy experiment, which is based on the use of a magnetic bottle time of flight spectrometer. These spectrometers were introduced at the beginning of the $X X I^{\text {st }}$ century and have allowed decisive progress in the study of multiple photoionization. We presented a rapid review of the discoveries they made possible. We illustrated more specifically the power and the sensitivity of this new technique on a specific example: the decay by emission of three Auger electrons of an inner shell hole in the $\mathrm{N}=2$ shell of argon. Although the $2 \mathrm{p}$ hole decay is a weak process with a probability of the order of only $2.10^{-3}$, we showed that it is possible to characterize it in detail. We found that the three electrons emission is mainly simultaneous in the $2 p$ hole case, in contrast with the decay of the $2 s$ hole which proceeds mainly by sequential Auger electron cascades.

\section{Acknowledgments}

The experiment was performed at SOLEIL Synchrotron (France) at the PLEIADES beam line, with the approval of the Soleil Peer Review Committee (Project No. 20141216). We are grateful to J. Bozek, A. R. Milosavljevic, C. Nicolas, E. Robert and PLEIADES team for help during the measurements and to SOLEIL staff for stable operation of the storage ring. M. A. K. and K.I. acknowledge the support of the Labex Plas@Par managed by the Agence Nationale de la Recherche, as part of the "Programme d'Investissements d'Avenir" under Reference No. ANR-11-IDEX-0004-02. This work has been financially supported by the Research Council for Natural Sciences and Engineering of the Academy of Finland.

\section{References}

[1] Briggs J S and Schmidt V 2000 Differential cross sections for photo-double-ionization of the helium atom J. Phys. B At. Mol. Opt. Phys. 33 R1-48

[2] Avaldi L and Huetz A 2005 Photodouble ionization and the dynamics of electron pairs in the continuum J. Phys. B At. Mol. Opt. Phys. 38 S861-91

[3] Viefhaus J, Snell G, Hentges R, Wiedenhöft M, Heiser F, Geßner O and Becker U 1998 Interference Effects between Auger and Photoelectron Studied by Subnatural Linewidth Auger-Photoelectron Coincidence Spectroscopy Phys. Rev. Lett. 80 1618-21 
[4] Viefhaus J, Cvejanović S, Langer B, Lischke T, Prümper G, Rolles D, Golovin A V, Grum-Grzhimailo A N, Kabachnik N M and Becker U 2004 Energy and Angular Distributions of Electrons Emitted by Direct Double Auger Decay Phys. Rev. Lett. 92

[5] Krassig B and Schmidt V 1992 Double-ZEKE coincidence spectroscopy J. Phys. B At. Mol. Opt. Phys. 25 L327

[6] Hall R I, Dawber G, McConkey A, MacDonald M A and King G C 1992 Vibrational structure of the $\mathrm{O} 22+$ ground state observed by threshold photoelectron coincidence spectroscopy Phys. Rev. Lett. 68 2751-4

[7] Penent F, Hall R I, Panajotović R, Eland J H D, Chaplier G and Lablanquie P 1998 New Method for the Study of Dissociation Dynamics of State-Selected Doubly Charged Ions: Application to CO $2+$ Phys. Rev. Lett. 81 3619-22

[8] Gisselbrecht M, Lavollée M, Huetz A, Bolognesi P, Avaldi L, Seccombe D P and Reddish T J 2006 Photodouble Ionization Dynamics for Fixed-in-Space H 2 Phys. Rev. Lett. 96

[9] Dörner R, Mergel V, Jagutzki O, Spielberger L, Ullrich J, Moshammer R and SchmidtBöcking H 2000 Cold Target Recoil Ion Momentum Spectroscopy: a "momentum microscope" to view atomic collision dynamics Phys. Rep. 330 95-192

[10] Arion T and Hergenhahn U 2015 Coincidence spectroscopy: Past, present and perspectives J. Electron Spectrosc. Relat. Phenom. 200 222-31

[11] Viefhaus J, Braune M, Korica S, Reinköster A, Rolles D and Becker U 2005 Auger cascades versus direct double Auger: relaxation processes following photoionization of the Kr 3d and Xe 4d, 3d inner shells J. Phys. B At. Mol. Opt. Phys. 38 3885-903

[12] Eland J H . 2003 Complete double photoionisation spectra of small molecules from TOFPEPECO measurements Chem. Phys. 294 171-86

[13] Eland J H D, Vieuxmaire O, Kinugawa T, Lablanquie P, Hall R I and Penent F 2003 Complete Two-Electron Spectra in Double Photoionization: The Rare Gases Ar, Kr, and Xe Phys. Rev. Lett. 90

[14] Beamson G, Porter H Q and Turner D W 1980 The collimating and magnifying properties of a superconducting field photoelectron spectrometer J. Phys. [E] 13 64-6

[15] Kruit P and Read F H 1983 Magnetic field paralleliser for $2 \pi$ electron-spectrometer and electron-image magnifier J. Phys. [E] 16 313-24

[16] Penent F, Palaudoux J, Lablanquie P, Andric L, Feifel R and Eland J H D 2005 Multielectron Spectroscopy: The Xenon 4 d Hole Double Auger Decay Phys. Rev. Lett. 95

[17] Hikosaka Y, Lablanquie P, Penent F, Kaneyasu T, Shigemasa E, Eland J H D, Aoto T and Ito K 2007 Single, double, and triple Auger decay of the Xe 4 p core-hole states Phys. Rev. A 76 
[18] Palaudoux J, Lablanquie P, Andric L, Ito K, Shigemasa E, Eland J H D, Jonauskas V, Kučas S, Karazija R and Penent F 2010 Multielectron spectroscopy: Auger decays of the krypton 3 d hole Phys. Rev. A 82

[19] Palaudoux J, Huttula S-M, Huttula M, Penent F, Andric L and Lablanquie P 2015 Auger decay paths of mercury $5 \mathrm{p}$ and $4 \mathrm{f}$ vacancies revealed by multielectron spectroscopy Phys. Rev. A 91

[20] Andersson E, Fritzsche S, Linusson P, Hedin L, Eland J H D, Rubensson J-E, Karlsson L and Feifel R 2010 Multielectron coincidence study of the double Auger decay of $3 \mathrm{~d}$ ionized krypton Phys. Rev. A 82

[21] Eland J H D, Rigby C F, Andersson E, Palaudoux J, Andric L, Penent F, Linusson P, Hedin L, Karlsson L, Rubensson J-E, Hikosaka Y, Ito K, Lablanquie P and Feifel R 2010 Spectra of the triply charged ion CS[sub 2][sup 3+] and selectivity in molecular Auger effects J. Chem. Phys. 132104311

[22] Eland J H D, Hochlaf M, Linusson P, Andersson E, Hedin L and Feifel R 2010 Triple ionization spectra by coincidence measurements of double Auger decay: The case of OCS J. Chem. Phys. 13214311

[23] Penent F, Lablanquie P, Palaudoux J, Andric L, Gamblin G, Hikosaka Y, Ito K and Carniato S 2011 Unveiling Residual Molecular Binding in Triply Charged Hydrogen Bromide Phys. Rev. Lett. 106

[24] Hikosaka Y, Aoto T, Lablanquie P, Penent F, Shigemasa E and Ito K 2006 Experimental Investigation of Core-Valence Double Photoionization Phys. Rev. Lett. 97

[25] Eland J H D, Linusson P, Hedin L, Andersson E, Rubensson J-E and Feifel R 2010 Triple ionisation of methane by double Auger and related pathways Chem. Phys. Lett. 485 21-5

[26] Niskanen J, Carravetta V, Vahtras O, Ågren H, Aksela H, Andersson E, Hedin L, Linusson P, Eland J H D, Karlsson L, Rubensson J-E and Feifel R 2010 Experimental and theoretical study of core-valence double photoionization of OCS Phys. Rev. A 82

[27] Eland J H D, Fink R F, Linusson P, Hedin L, Plogmaker S and Feifel R 2011 Single and multiple photoionisation of H2S by 40-250 eV photons Phys. Chem. Chem. Phys. 1318428

[28] Niskanen J, Andersson E, Eland J H D, Linusson P, Hedin L, Karlsson L, Feifel R and Vahtras O 2012 Symmetry breaking in core-valence double photoionization of SO 2 Phys. Rev. A 85

[29] Huttula S-M, Lablanquie P, Andric L, Palaudoux J, Huttula M, Sheinerman S, Shigemasa E, Hikosaka Y, Ito K and Penent F 2013 Decay of a 2 p Inner-Shell Hole in an Ar + Ion Phys. Rev. Lett. 110

[30] Gottwald A, Gerth C and Richter M 19994 d Photoionization of Free Singly Charged Xenon Ions Phys. Rev. Lett. 82 2068-70 
[31] Bizau J-M, Cubaynes D, Guilbaud S, Penent F, Lablanquie P, Andric L, Palaudoux J, Al Shorman M M and Blancard C 2016 Photoelectron Spectroscopy of Ions: Study of the Auger Decay of the $4 \mathrm{~d} \rightarrow \mathrm{nf}(\mathrm{n}=4,5)$ Resonances in Xe $5+$ Ion Phys. Rev. Lett. 116

[32] Andersson E, Linusson P, Fritzsche S, Hedin L, Eland J H D, Karlsson L, Rubensson J-E and Feifel R 2012 Formation of Kr 3 + via core-valence doubly ionized intermediate states Phys. Rev. A 85

[33] Huttula M, Huttula S-M, Fritzsche S, Lablanquie P, Penent F, Palaudoux J and Andric L 2014 Core-valence double photoionization of atomic mercury Phys. Rev. A 89

[34] Huttula S-M, Soronen J, Huttula M, Penent F, Palaudoux J, Andric L and Lablanquie P 2015 Auger decay of core valence double photoionized states in atomic mercury $J$. Phys. B At. Mol. Opt. Phys. 48115001

[35] Eland J H D, Linusson P, Hedin L, Andersson E, Rubensson J-E and Feifel R 2008 Coincidence technique using synchrotron radiation for triple photoionization: Results on rare gas atoms Phys. Rev. A $\mathbf{7 8}$

[36] Hikosaka Y, Lablanquie P, Penent F, Kaneyasu T, Shigemasa E, Feifel R, Eland J H D and Ito K 2009 Energy Correlation of the Three Electrons Emitted during the Triple Photoionization of Ar Phys. Rev. Lett. 102

[37] Hikosaka Y, Lablanquie P, Penent F, Palaudoux J, Andric L, Soejima K, Shigemasa E, Suzuki I H, Nakano M and Ito K 2011 Energy Correlation among Three Photoelectrons Emitted in Core-Valence-Valence Triple Photoionization of Ne Phys. Rev. Lett. 107

[38] Mucke M, Braune M, Barth S, Förstel M, Lischke T, Ulrich V, Arion T, Becker U, Bradshaw A and Hergenhahn U 2010 A hitherto unrecognized source of low-energy electrons in water Nat. Phys. 6 143-6

[39] Lablanquie P, Penent F and Hikosaka Y 2016 Multi-electron coincidence spectroscopy: double photoionization from molecular inner-shell orbitals J. Phys. B At. Mol. Opt. Phys. 49182002

[40] Lablanquie P, Penent F, Palaudoux J, Andric L, Selles P, Carniato S, Bučar K, Žitnik M, Huttula M, Eland J H D, Shigemasa E, Soejima K, Hikosaka Y, Suzuki I H, Nakano M and Ito K 2011 Properties of Hollow Molecules Probed by Single-Photon Double Ionization Phys. Rev. Lett. 106

[41] Lablanquie P, Grozdanov T P, Žitnik M, Carniato S, Selles P, Andric L, Palaudoux J, Penent F, Iwayama H, Shigemasa E, Hikosaka Y, Soejima K, Nakano M, Suzuki I H and Ito K 2011 Evidence of Single-Photon Two-Site Core Double Ionization of C 2 H 2 Molecules Phys. Rev. Lett. 107

[42] Nakano M, Penent F, Tashiro M, Grozdanov T P, Žitnik M, Carniato S, Selles P, Andric L, Lablanquie P, Palaudoux J, Shigemasa E, Iwayama H, Hikosaka Y, Soejima K, Suzuki I H, Kouchi $\mathrm{N}$ and Ito K 2013 Single Photon $\mathrm{K}-2$ and $\mathrm{K}-1 \mathrm{~K}-1$ Double Core Ionization in C 2 H $2 \mathrm{n}(\mathrm{n}=1-3), \mathrm{CO}$, and N 2 as a Potential New Tool for Chemical Analysis Phys. Rev. Lett. 110 
[43] Penent F, Nakano M, Tashiro M, Grozdanov T P, Žitnik M, Carniato S, Selles P, Andric L, Lablanquie P, Palaudoux J, Shigemasa E, Iwayama H, Hikosaka Y, Soejima K, Suzuki I H, Kouchi N and Ito K 2014 Molecular single photon double K-shell ionization J. Electron Spectrosc. Relat. Phenom. 196 38-42

[44] Hedin L, Tashiro M, Linusson P, Eland J H D, Ehara M, Ueda K, Zhaunerchyk V, Karlsson L and Feifel R 2014 Single site double core level ionisation of OCS Chem. Phys. 439 111-6

[45] Hedin L, Tashiro M, Linusson P, Eland J H D, Ehara M, Ueda K, Zhaunerchyk V, Karlsson L, Pernestål K and Feifel R 2014 N1s and O1s double ionization of the NO and N2O molecules J. Chem. Phys. 14044309

[46] Penent F, Nakano M, Tashiro M, Grozdanov T P, Žitnik M, Bučar K, Carniato S, Selles P, Andric L, Lablanquie P, Palaudoux J, Shigemasa E, Iwayama H, Hikosaka Y, Soejima K, Suzuki I H, Berrah N, Wuosmaa A H, Kaneyasu T and Ito K 2015 Double core hole spectroscopy with synchrotron radiation J. Electron Spectrosc. Relat. Phenom. 204 303-12

[47] Tashiro M, Nakano M, Ehara M, Penent F, Andric L, Palaudoux J, Ito K, Hikosaka Y, Kouchi N and Lablanquie P 2012 Auger decay of molecular double core-hole and its satellite states: Comparison of experiment and calculation J. Chem. Phys. 137224306

[48] Nakano M, Selles P, Lablanquie P, Hikosaka Y, Penent F, Shigemasa E, Ito K and Carniato S 2013 Near-Edge X-Ray Absorption Fine Structures Revealed in Core Ionization Photoelectron Spectroscopy Phys. Rev. Lett. 111

[49] Carniato S, Selles P, Andric L, Palaudoux J, Penent F, Žitnik M, Bučar K, Nakano M, Hikosaka Y, Ito K and Lablanquie P 2015 Single photon simultaneous K-shell ionization and K-shell excitation. I. Theoretical model applied to the interpretation of experimental results on $\mathrm{H} 2 \mathrm{O}$ J. Chem. Phys. 14214307

[50] Carniato S, Selles P, Andric L, Palaudoux J, Penent F, Žitnik M, Bučar K, Nakano M, Hikosaka Y, Ito K and Lablanquie P 2015 Single photon simultaneous K-shell ionization and K-shell excitation. II. Specificities of hollow nitrogen molecular ions J. Chem. Phys. 14214308

[51] Carniato S, Selles P, Lablanquie P, Palaudoux J, Andric L, Nakano M, Hikosaka Y, Ito K, Marchenko T, Travnikova O, Goldsztejn G, Journel L, Guillemin R, Céolin D, Simon M, Piancastelli M N and Penent F 2016 Photon-energy dependence of single-photon simultaneous core ionization and core excitation in CO 2 Phys. Rev. A 94

[52] Lablanquie P, Huttula S-M, Huttula M, Andric L, Palaudoux J, Eland J H D, Hikosaka Y, Shigemasa E, Ito K and Penent F 2011 Multi-electron spectroscopy: Auger decays of the argon 2s hole Phys. Chem. Chem. Phys. 1318355

[53] Lablanquie P, Andric L, Palaudoux J, Becker U, Braune M, Viefhaus J, Eland J H D and Penent F 2007 Multielectron spectroscopy: Auger decays of the argon 2p hole J. Electron Spectrosc. Relat. Phenom. 156-158 51-7 
[54] Palaudoux J, Sheinerman S, Soronen J, Huttula S-M, Huttula M, Jänkälä K, Andric L, Ito K, Lablanquie P, Penent F, Bizau J-M, Guilbaud S and Cubaynes D 2015 Valence Auger decay following $3 \mathrm{~s}$ photoionization in potassium Phys. Rev. A 92

[55] Liu X-J, Miao Q, Gel'mukhanov F, Patanen M, Travnikova O, Nicolas C, Ågren H, Ueda K and Miron C 2014 Einstein-Bohr recoiling double-slit gedanken experiment performed at the molecular level Nat. Photonics 9 120-5

[56] Mehlhorn W 1960 Die Feinstruktur desL-MM-Auger-Elektronenspektrums von Argon und derK-LL-Spektren von Stickstoff, Sauerstoff und Methan Z. F pr Phys. 160 247-67

[57] Pulkkinen H, Aksela S, Sairanen O-P, Hiltunen A and Aksela H 1996 Correlation effects in the - MM Auger transitions of Ar J. Phys. B At. Mol. Opt. Phys. 29 3033-50

[58] Carlson T A and Krause M O 1965 Experimental Evidence for Double Electron Emission in an Auger Process Phys. Rev. Lett. 14 390-2

[59] Ricz S, Kövér á., Jurvansuu M, Varga D, Molnár J and Aksela S 2002 High-resolution photoelectron-Auger-electron coincidence study for the L 23-M 23 M 23 transitions of argon Phys. Rev. A $\mathbf{6 5}$

[60] Kramida, A., Ralchenko, Yu., Reader, J. and NIST ASD Team (2015) NIST Atomic Spectra Database (ver. 5.3), [Online]. Available: http://physics.nist.gov/asd [2016, July 21]. National Institute of Standards and Technology, Gaithersburg, MD

[61] King G C, Tronc M, Read F H and Bradford R C 1977 An investigation of the structure near the $\mathrm{L}_{2,3}$ edges of argon, the $\mathrm{M}_{4,5}$ edges of krypton and the $\mathrm{N}_{4,5}$ edges of xenon, using electron impact with high resolution J. Phys. B At. Mol. Phys. 10 2479-95

[62] Dalitz R H 1953 CXII. On the analysis of $\tau$-meson data and the nature of the $\tau$-meson Lond. Edinb. Dublin Philos. Mag. J. Sci. 44 1068-80

[63] Arp U, LeBrun T, Southworth S H, MacDonald M A and Jung M 1997 X-ray fluorescence and Auger-electron coincidence spectroscopyof vacancy cascades in atomic argon $P$ hys. Rev. A 55 4273-84

[64] Müller A, Borovik A, Buhr T, Hellhund J, Holste K, Kilcoyne A L D, Klumpp S, Martins M, Ricz S, Viefhaus J and Schippers S 2015 Observation of a Four-Electron Auger Process in Near- K -Edge Photoionization of Singly Charged Carbon Ions Phys. Rev. Lett. 114

[65] Glans P, LaVilla R E, Ohno M, Svensson S, Bray G, Wassdahl N and Nordgren J 1993 Determination of the lifetime width of the argon L 1 -hole state Phys. Rev. A 47 1539-42 


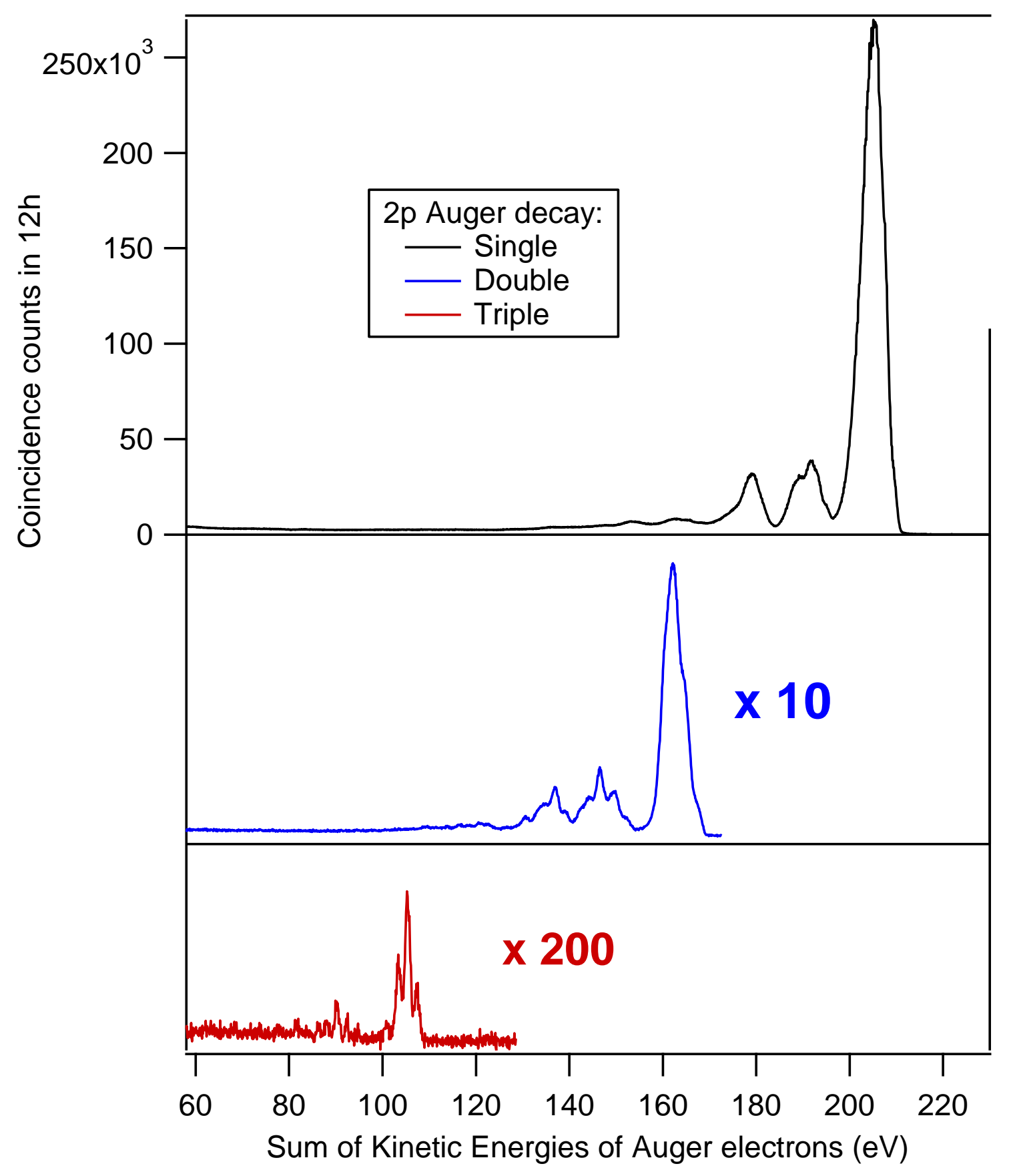

Fig 1: Auger spectra for the Auger decay of an Ar $2 p$ hole by emission of one, two or three Auger electrons. The Auger spectra are represented as a function of the total kinetic energy of the Auger electron(s) (respectively, 1, 2 or 3) and visualize the final sate populations. They have been obtained respectively from 2, 3 and 4 electron coincidence events where a $2 p$ photoelectron is detected in coincidence with 1,2 or 3 other electrons. A bin size of $100 \mathrm{meV}$ was used here. 


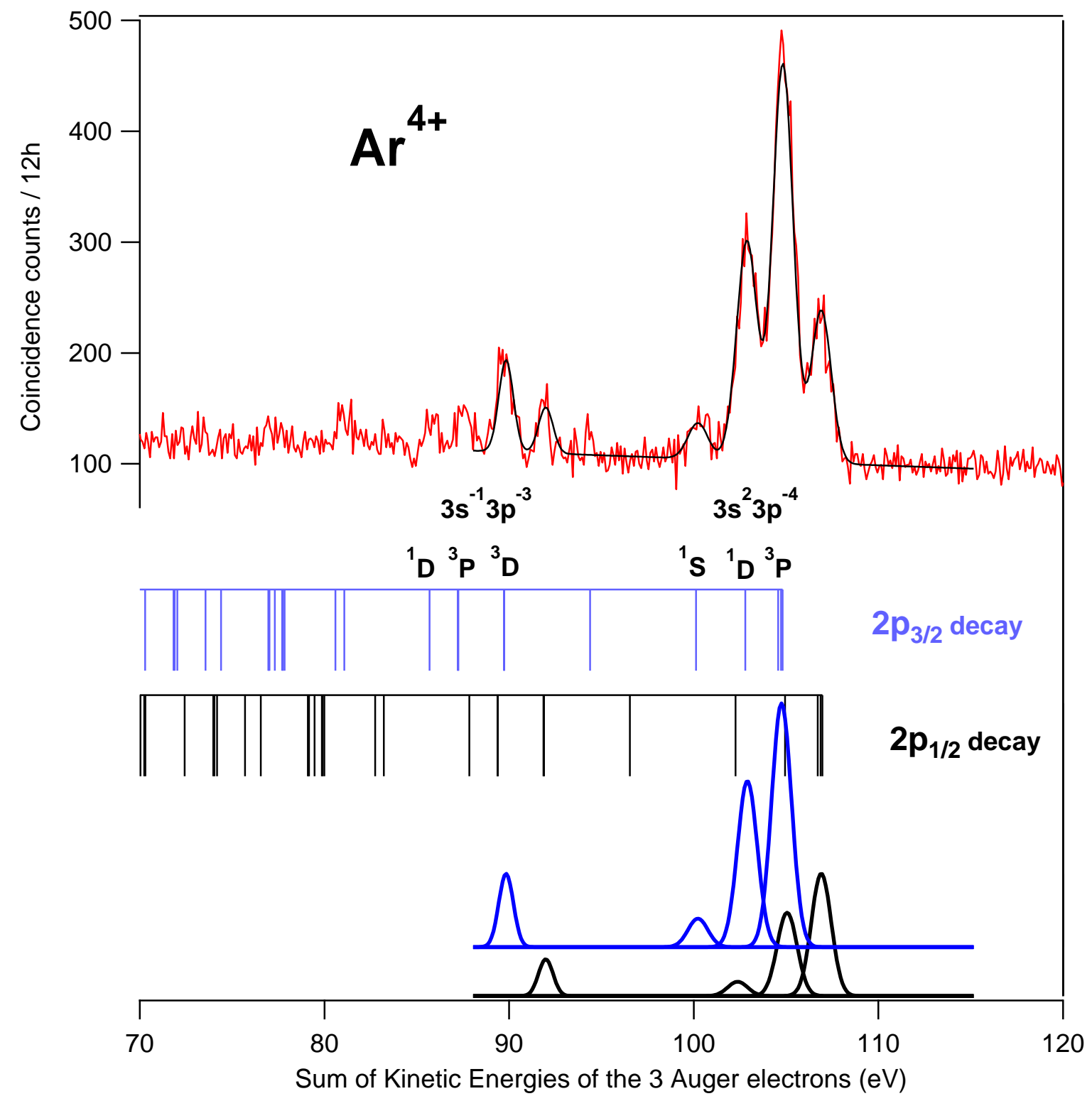

Fig 2: Triple Auger decay of an $\operatorname{Ar} 2 p$ hole: enlargement of the triple Auger spectrum at the bottom of Fig 1. The peaks reflect the transitions from the $2 p$ hole $\left(2 p_{3 / 2}\right.$ or $\left.2 p_{1 / 2}\right)$ to the final $\mathrm{Ar}^{4+}$ states. The vertical bars indicate the expected position, obtained from the $\mathrm{Ar}^{4+}$ levels tabulated in the NIST tables [60]. A fit of the spectrum is shown; it was obtained by imposing a statistical ratio of $2 / 1$ for the intensities of the transitions from $2 p_{3 / 2} / 2 p_{1 / 2}$ core holes, and by using the tabulated $\mathrm{Ar}^{4+}$ energy spacings [60]. 


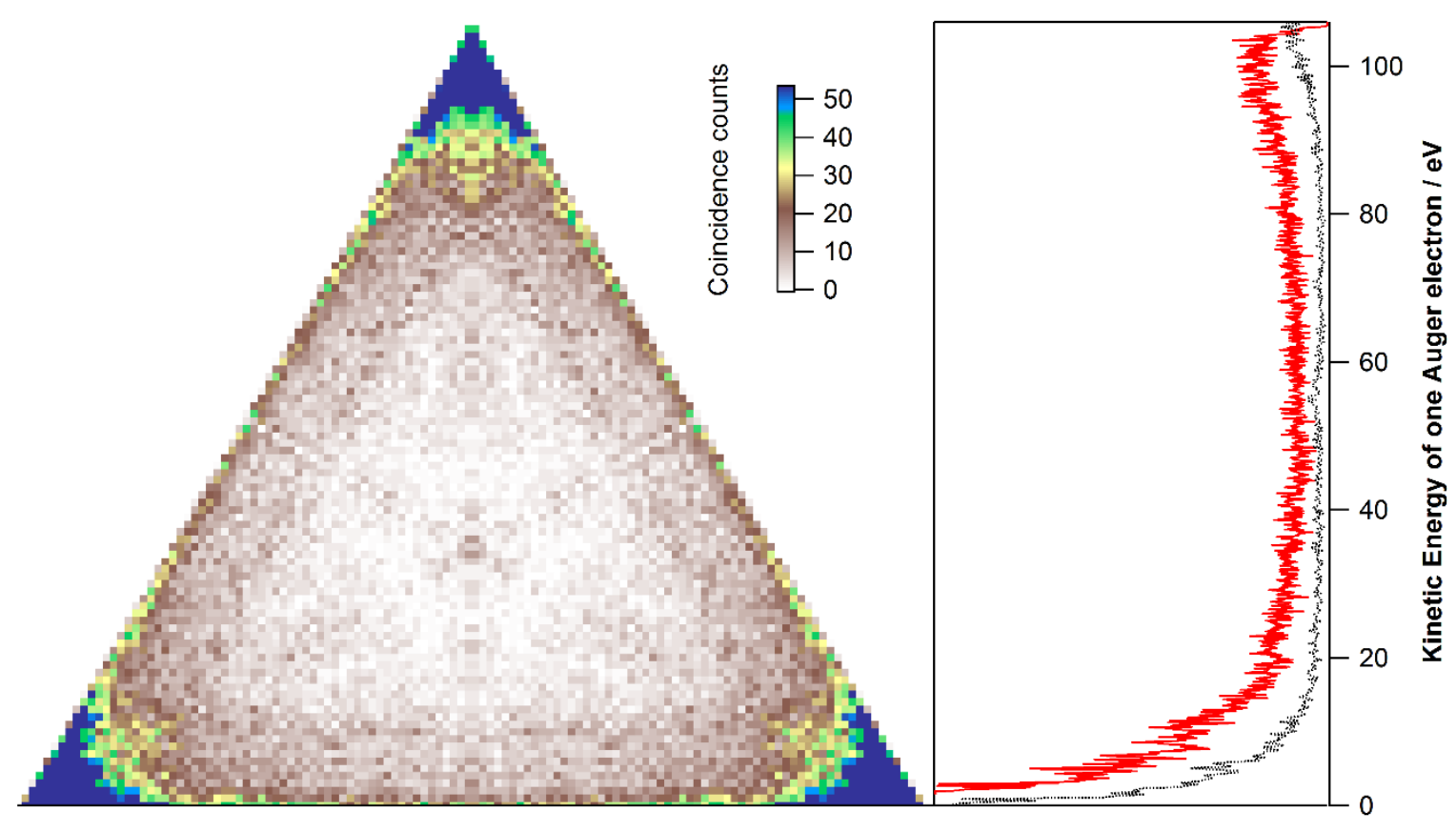

Fig 3 : Triple Auger decay of an $\operatorname{Ar} 2 p$ hole. Left shows the Dalitz plot displaying the energy correlation between the three Auger electrons detected in coincidence with a $2 p$ photoelectron, and with the constraint that a final $\mathrm{Ar}^{4+} 3 \mathrm{p}^{-4}$ level is reached (sum of the kinetic energy of the three Auger electrons is imposed to lie in the $102-107.7 \mathrm{eV}$ energy range). The spectrum in the right (red) shows the energy of one of these three Auger electrons. It is obtained by the projection of the Dalitz triangle onto its perpendicular height. The black dotted curve gives an estimate of the background contribution, obtained by selecting an energy range of $110-115.7$ $\mathrm{eV}$ for the sum of the three electrons coincident with a $2 \mathrm{p}$ photoelectron 


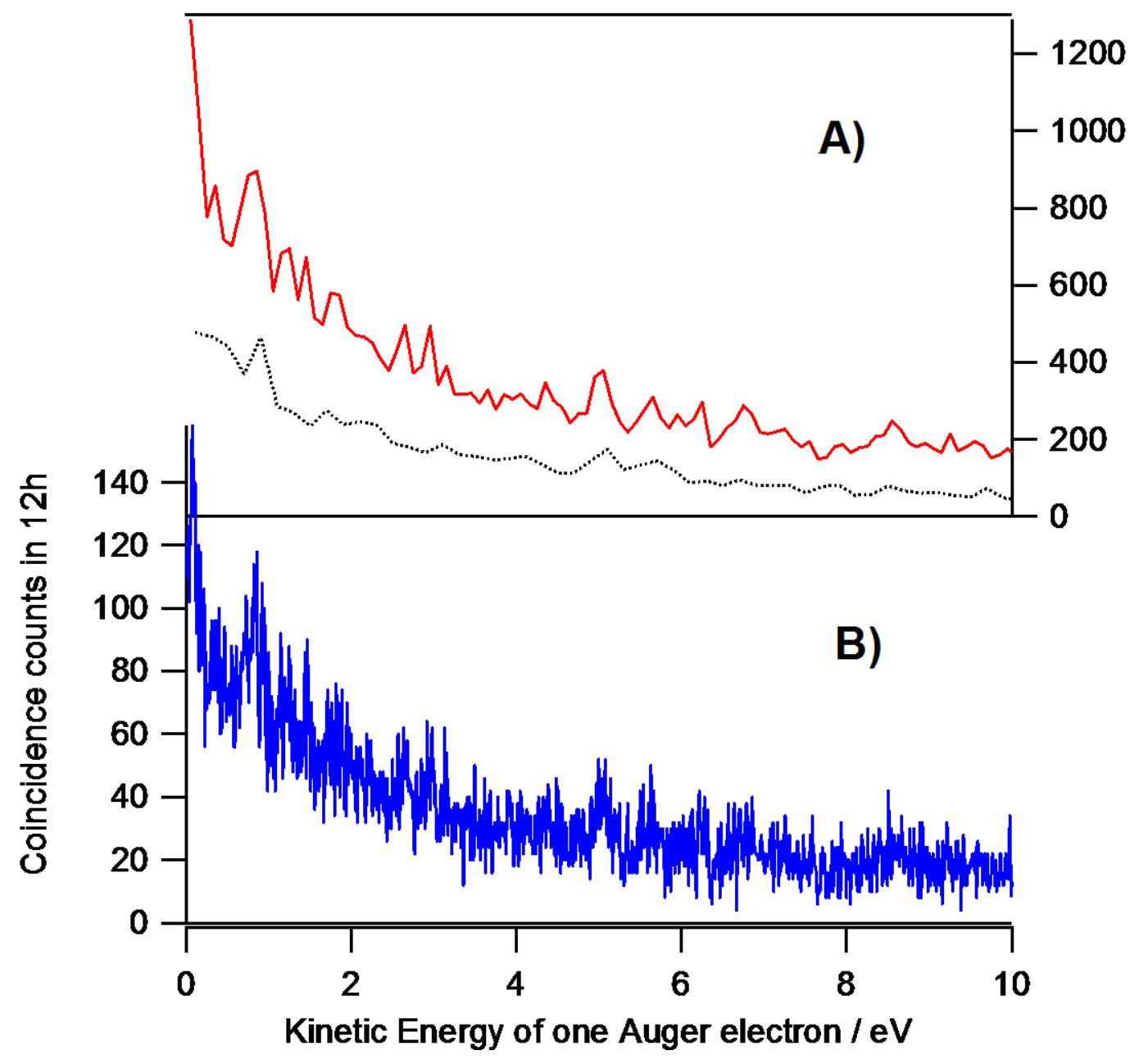

Fig 4: Low energy part of the distribution of energy of one of the Auger electrons released in the triple Auger decay of the $\operatorname{Ar} 2 p$ hole yielding $\mathrm{Ar}^{4+} 3 p^{-4}$ final states. A) is an enlargement of Fig 3 (right), obtained with a $100 \mathrm{meV}$ bin size; the black dotted curve shows the estimated background contribution. B) is a replot of the same data as in A), but with a discretization step of $10 \mathrm{meV}$, giving hints of structures due to the formation of excited $\mathrm{Ar}^{3+}$ states converging to excited $\mathrm{Ar}^{4+}$ states, and autoionizing to lower lying $\mathrm{Ar}^{4+}$ levels 


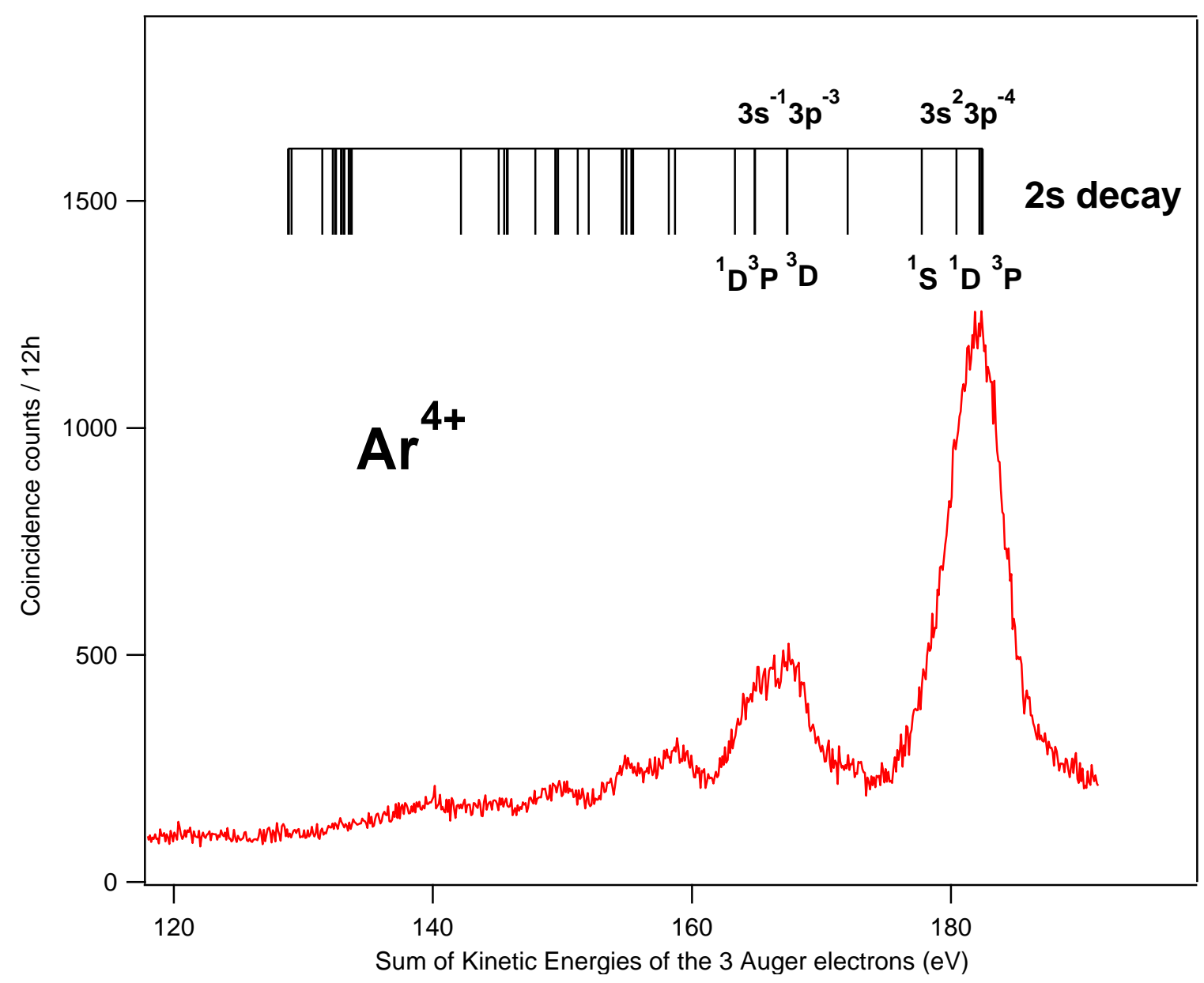

Fig 5: Triple Auger decay of an Ar 2s hole (equivalent of Fig 2 for the $2 p$ case). The triple Auger spectrum is obtained from the sum of the three (Auger) electrons detected with a $2 \mathrm{~s}$ photoelectron in all 4-electron coincidence events. The peaks reflect the transitions from the $2 \mathrm{~s}$ hole to the final $\mathrm{Ar}^{4+}$ states. The vertical bars indicate the expected position, obtained from the $\mathrm{Ar}^{4+}$ levels tabulated in the NIST tables. Note that the structure of the peaks is less well resolved than in Fig 2, partly because of the higher kinetic energies involved here, but essentially due to the shorter lifetime of the $2 \mathrm{~s}$ hole compared to the $2 \mathrm{p}$ ones that induces a $2.3 \mathrm{eV}$ lifetime broadening of the Auger spectrum. A bin size of $100 \mathrm{meV}$ was used here. 

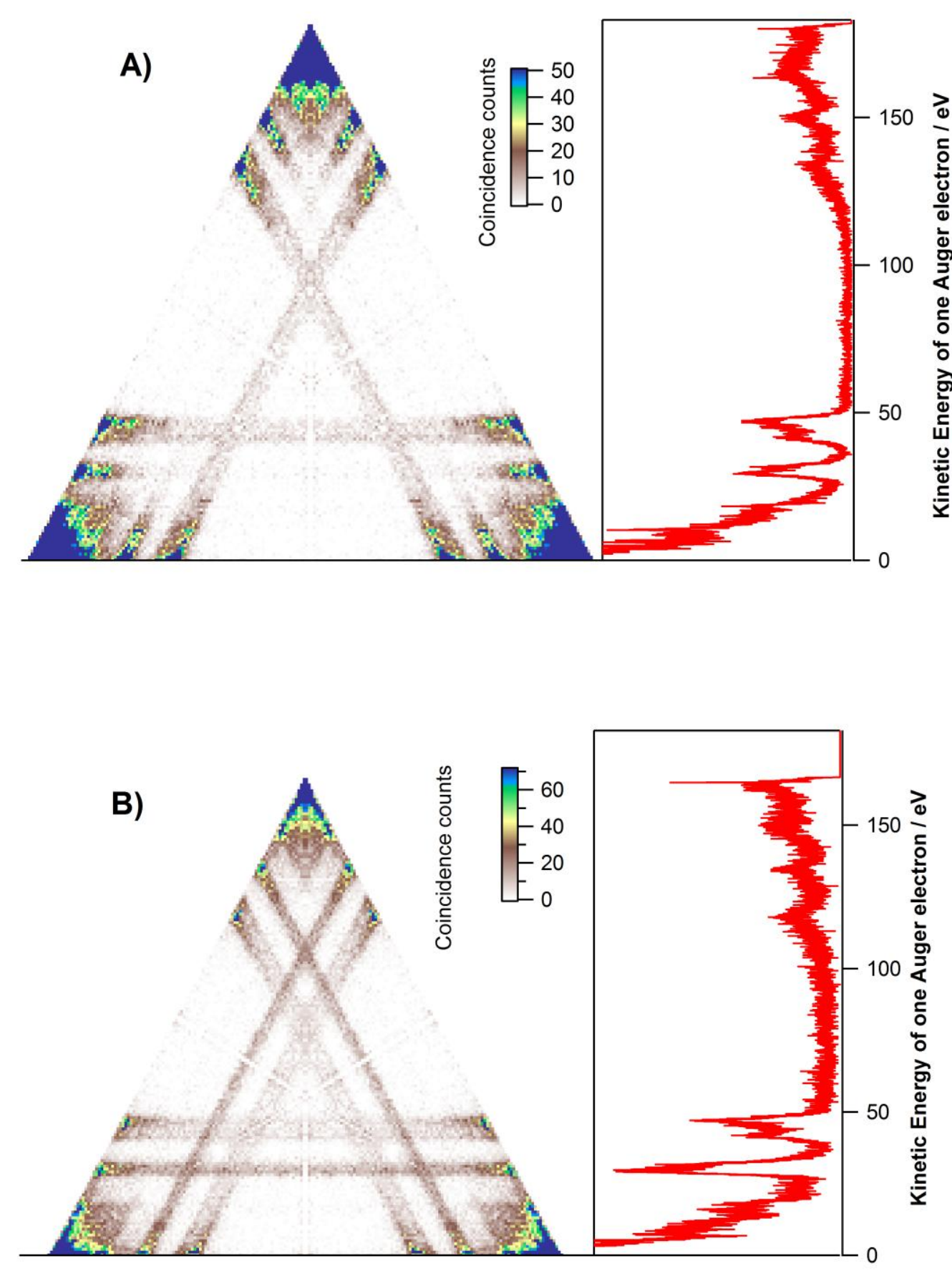

Fig 6 : Triple Auger decay of an $\operatorname{Ar} 2$ s hole, similar to Fig 3 for the case of the $2 p$ decay. A) shows the Dalitz plot and the energy of one of the Auger electrons when a final $\mathrm{Ar}^{4+} 3 \mathrm{p}^{-4}$ level is reached (sum of the kinetic energy of the three Auger electrons is imposed to lie in the 176 $186.5 \mathrm{eV}$ energy range). In $\mathrm{B}$ ) a final $\mathrm{Ar}^{4+} 3 \mathrm{~s}^{-1} 3 \mathrm{p}^{-3}$ level is reached (sum of the kinetic energy of the three Auger electrons is here imposed to lie in the $163.5-169 \mathrm{eV}$ energy range). NOTE : this figure is similar to Fig 7 in Lablanquie et al [52] but here statistics is better and dead time is shorter, revealing more clearly the direct emission of the two Auger electrons released in the last step of the Auger cascade. 